\title{
Estudio de diferentes metodologías para determinar la biodisponibilidad de cadmio y arsénico en suelos y su relación con la concentración en plantas
}

\author{
Olga Lucía Barragán Moreno \\ Licenciada en Química. Estudios de Maestría en Docencia de la Química. \\ Docente Universidad Jorge Tadeo Lozano y Gimnasio Moderno \\ Correspondencia: formaldehido1@gmail.com \\ Recibido: 08-02-08 / Aceptado 14-03-08
}

\begin{abstract}
Resumen
Aunque se han realizado trabajos en estandarización analítica de metodologías para evaluación de metales, aún quedan serías dudas en cuanto a la relación entre la efectividad de los métodos usados y la capacidad de las plantas para absorber dichos metales. Este parámetro permitiría definir en forma más clara la biodisponibilidad de metales y el riesgo de contaminación de cultivos. El presente artículo muestra los resultados de un trabajo de investigación realizado en Corpoica-Tibaitatá, cuyo objetivo fue determinar las concentraciones disponibles de cadmio y arsénico en el suelo y establecer una relación con las cantidades de éstos absorbidas por las plantas, encontrándose que las cantidades de estos metales, que se hallaban ligadas a los óxidos de hierro y manganeso, presentaban una relación directa con las cantidades absorbidas por ellas.

Palabras clave: absorción, biodisponibilidad, metales pesados, método de Tessier, toxicidad.
\end{abstract}

\begin{abstract}
A study of various methodologies to determine the bio-availability of cadmium and arsenic in soil and their relative concentration in plants

Although works have been made in analytical standardization of methodologies for metal evaluation, there are still major doubts as far as the effectiveness of the used methods and the capacity of the plants to absorb these metals. This parameter would allow defining in clearer form the bioavailability of metals and the risk of contamination of crops. This article shows the results of a research made in Corpoica-Tibaitatá, which objective was to determine the available concentrations of cadmium and arsenic in the ground and to establish a relation with the amounts of these absorbed by the plants; where it was found that the amounts of these metals, that were bound to oxides of iron and manganese, displayed a direct relation with the amounts absorbed by them.

Key words: absorption, bioavailability, heavy metals, method of Tessier, toxicity.
\end{abstract}




\section{Introducción}

En Colombia, como en otros países del mundo, cada día hay mayor conciencia respecto a la necesidad de proteger el medio ambiente y los recursos naturales esenciales para la supervivencia humana. Debido al uso continuo de las aguas del río Bogotá y sus afluentes, con fines de riego, en la sabana de Bogotá se presenta un alto porcentaje de contaminación de suelos y cultivos, particularmente en las zonas hortícolas (1).

Se ha determinado que las principales causas del alto grado de contaminación del río Bogotá y sus afluentes son: las continuas descargas de desechos y basuras urbanas e industriales, las aguas negras, los fertilizantes, las enmiendas y los agroquímicos. Estas sustancias entran al río por medio de las aguas de drenaje, trayendo como consecuencia aumento en el contenido de diferentes contaminantes, entre ellos los metales pesados, los cuales, muchas veces, sobrepasan los niveles críticos permisibles para las aguas de riego (1).

Generalmente las determinaciones sobre la contaminación de elementos pesados en suelos se realizan usando el llamado método convencional, el cual usa ácidos fuertes para la extracción, y sólo tiene en cuenta la concentración total. Este método tiene sus limitaciones, ya que es posible que solo las fracciones móviles de los metales pesados en el suelo sean las susceptibles de ser absorbidas por las plantas y por lo tanto puedan ser consideradas biodisponibles (2-5).

Llama la atención los resultados obtenidos por Gonzáles y Mejia, quienes encontraron que al aumentar las concentraciones de cadmio (Cd) y arsénico (As), a través del agua de riego, el rendimiento de las hortalizas usadas para el estudio disminuyó considerablemente (6). Al determinar las concentraciones de estos metales absorbidos por las plantas (lechuga, zanahoria y pepino), estas eran mayores a los permitidas por la Organización Mundial de la Salud (OMS) $(7,8)$. Teniendo presente este panorama, se consideró necesario estandarizar una metodología que permitiera determinar las fracciones móviles de estos metales.

\section{Metodología}

Se utilizaron procedimientos donde la fase móvil de los metales pesados en suelos, (aquella relacionada con adsorciones débiles, metal intercambiable, asociados a óxidos de hierro $(\mathrm{Fe})$ y manganeso $(\mathrm{Mn})$, materia orgánica y carbonatos), se extraen y son comparadas con los contenidos totales tanto en los suelos como en los cultivos. Se considera que estas mediciones permiten una mejor interpretación del potencial de biodisponibilidad de estos elementos en el suelo.

Se tomaron 7 muestras de suelo del primer horizonte $(0-15 \mathrm{~cm})$ de tres municipios aledaños al río Bogotá: Bosa, Cota y Ricaurte, Cundinamarca, Colombia. Según el Instituto Geográfico Agustín Codazzi, estos suelos tienen como clasificación taxonómica: Fluventic Tropaquepts, Dystric Haplustand y Fluventic Ustropepts, respectivamente.

Los suelos fueron caracterizados de acuerdo con las metodologías utilizadas en el laboratorio de química de suelos de Corpoica - Tibaitatá (9). Para la extracción convencional de $\mathrm{Cd}$ y As se empleó una mezcla de ácidos fluorhídrico $(\mathrm{HF})$, clorhídrico $(\mathrm{HCl})$ y perclórico $\left(\mathrm{HClO}_{4}\right)$ concentrados en una relación 1:1:1, en frío. Dado que en las pruebas preliminares se obtuvieron mejores resultados cuando se empleo $\mathrm{HClO}_{4}$ que con el ácido nítrico $\left(\mathrm{HNO}_{3}\right)$ se decidió seguir usando $\mathrm{HClO}_{4}$.

Para la extracción de la fase móvil o disponible de $\mathrm{Cd}$ y As se emplearon ácido acético $\left(\mathrm{CH}_{3} \mathrm{COOH}\right) 0,43 \mathrm{M}$, nitrato cálcico $\left(\mathrm{Ca}\left(\mathrm{NO}_{3}\right)_{2}\right)$ 0,1M, $\mathrm{HCl} 0,1 \mathrm{M}$ y EDTA $0,02 \mathrm{M}$ en acetato de amonio $\left(\mathrm{CH}_{3} \mathrm{COONH}_{4}\right) 0,03 \mathrm{M}$ y el método de Tessier. Usando este último método se obtienen 6 fracciones de metales:

1. Fracción en solución acuosa del suelo.

2. Fracción intercambiable.

3. Fracción asociada a carbonatos.

4. Fracción asociada a óxidos de Fe y Mn.

5. Fracción asociada a materia orgánica.

6. Fracción asociada a silicatos o residual (10).

Para el ensayo de invernadero, de los suelos muestreados se tomaron $2 \mathrm{Kg}$, los cuales se colocaron en bolsas de polietileno, en cada una de ellas se transplantó una lechuga (variedad Black Seeded Simpson), de un semillero previamente realizado con la misma clase de suelo. Estas lechugas se recolectaron a los 60 días y se sometieron a una digestión ácida siguiendo la metodología utilizada en el laboratorio de química de suelos de Corpoica - Tibaitatá (9). Las concentraciones de cadmio se determinaron por 
Tabla 1. Caracterización de los suelos

\begin{tabular}{|c|c|c|c|c|c|c|c|c|c|c|c|c|c|c|c|c|c|}
\hline LUGAR & No. & & ALISIS & TEXTL & JRAL & & M.O & $\mathrm{Ca}$ & Mg & $K$ & $\mathrm{Na}$ & CICE & $P$ & Cd & As & $\mathrm{Fe}$ & Mn \\
\hline ORIGEN & SUELO & \%A & $\% A r$ & $\% \mathrm{~L}$ & TEXT & & (\%) & \multicolumn{5}{|c|}{$\mathrm{Cmol}+/ \mathrm{Kg}$} & \multicolumn{5}{|c|}{$\mathrm{mg} / \mathrm{Kg}$} \\
\hline Bosa & 1 & 38.3 & 38.4 & 23.4 & FrAr & 5.9 & 8.0 & 16.3 & 2.2 & 0.25 & 1.50 & 20.3 & 67.0 & 3.35 & 0.98 & 130 & $\overline{23.2}$ \\
\hline osa & 2 & 10.3 & 74.4 & 15.4 & $\mathrm{Ar}$ & 5.3 & 3.1 & 15.0 & 4.2 & 1.27 & 1.30 & 21.8 & 80.0 & 3.09 & 1.69 & 240 & 17.1 \\
\hline Cota & 3 & 34.3 & 22.4 & 43.4 & $\mathrm{Fr}$ & 6.7 & 6.0 & 16.0 & 2.7 & 0.82 & 0.97 & 20.5 & 46.0 & 4.73 & 1.10 & 49 & 18 \\
\hline Cota & 4 & 29.3 & 24.4 & 46.4 & $\mathrm{Fr}$ & 6.5 & 7.7 & 20.6 & 2.8 & 1.34 & 1.08 & 25.8 & 83.0 & 2.58 & 1.20 & 77 & 19.3 \\
\hline Cota & 5 & 34.6 & 22.4 & 43.0 & $\mathrm{Fr}$ & 6.6 & 7.3 & 20.1 & 2.0 & 0.51 & 0.92 & 23.5 & 60.0 & 3.01 & 1.78 & 470 & 8.8 \\
\hline Cota & 6 & 30.6 & 26.4 & 43.0 & $\mathrm{Fr}$ & 6.5 & 6.8 & 18.0 & 1.4 & 1.15 & 0.64 & 21.2 & 66.0 & 3.10 & 2.54 & 480 & 19 \\
\hline caurte & 7 & 27.6 & 42.4 & 30.0 & $\mathrm{Ar}$ & 6.7 & 2.8 & 19.2 & 3.6 & 0.88 & 0.49 & 24.2 & 79.0 & 4.39 & 2.54 & 120 & 10.5 \\
\hline
\end{tabular}

$A=\operatorname{arena} ; \mathrm{Ar}=$ arcilla; $\mathrm{L}=$ limo; $\mathrm{Fr}=$ franco; $\mathrm{Ar}=$ Arcilloso; M.O.= Materia orgánica

Tabla 2. Concentraciones de Cadmio y Arsénico en mg/Kg absorbidas por las plantas

\begin{tabular}{ccc}
\hline SUELO & CADMIO & ARSÉNICO \\
\hline 1 & 0.093 & 0.116 \\
2 & 0.197 & 0.127 \\
3 & 0.082 & 0.186 \\
4 & 0.059 & 0.171 \\
5 & 0.079 & 0.156 \\
6 & 0.044 & 0.148 \\
7 & 0.075 & 0.199 \\
\hline
\end{tabular}

horno de grafito y llama, y las de arsénico por generación de hidruros en un espectrofotómetro de absorción (Perkin Elmer modelo 2083).

\section{Resultados}

La caracterización de los suelos empleados se muestra en la Tabla 1. Se observa que son suelos fértiles, con altos contenidos de fósforo $(\mathrm{P})$ y bases intercambiables (macronutrientes), tienen altos valores de capacidad de intercambio catiónico efectivo (CICE) debido a los contenidos de materia orgánica y arcilla. Para los metales pesados $\mathrm{Cd}$ y As se determinó un contenido total alto.

En la Tabla 2 se muestran las concentraciones de $\mathrm{Cd}$ y As encontradas en los suelos objeto de estudio.

En la Figura 1, se muestra la relación entre los contenidos biodisponibles de Cd extraídos de los suelos analizados con $\mathrm{Ca}\left(\mathrm{NO}_{3}\right)_{2}$ y la concentración respectiva de $\mathrm{Cd}$ en las plantas.

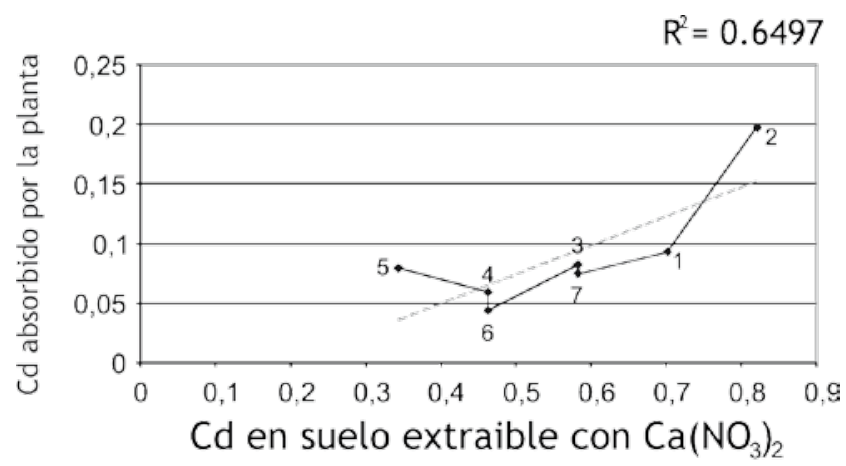

Figura 1. Relación entre las concentraciones biodisponibles $(\mathrm{mg} / \mathrm{kg})$ de $\mathrm{Cd}$ extraidas con $\mathrm{Ca}(\mathrm{NO} 3) 2$ y la cantidad de este metal $(\mathrm{mg} / \mathrm{kg})$ absorbidos por la planta.

En la Figura 2 se muestra la relación entre la absorción de Cd por la planta y la concentración de este unido a óxidos de Fe y Mn (a) y a la materia orgánica (b) de acuerdo con el método de Tessier. Se observa que al aumentar el contenido de $\mathrm{Cd}$ asociado a óxidos de Fe y Mn y a materia orgánica existe una tendencia a disminuir la cantidad de Cd absorbido por la planta, lo cual, de acuerdo con lo planteado por Anderson y Hahlin, existe afinidad de los ácidos húmicos y fúlvicos por este elemento y la superficie de los óxidos muestran una selectividad hacia la adsorción de diversos metales (13). Con el As no se evidenció esta relación.

\section{Discusión}

Es importante anotar que los contenidos totales en suelos superiores a $3 \mathrm{mg} / \mathrm{Kg}$ para Cd y $0.03 \mathrm{mg} / \mathrm{Kg}$ para As se consideran fitotóxicos según datos suministrados por la OMS $(7,8,11,12)$. Lo anterior se puede relacionar 


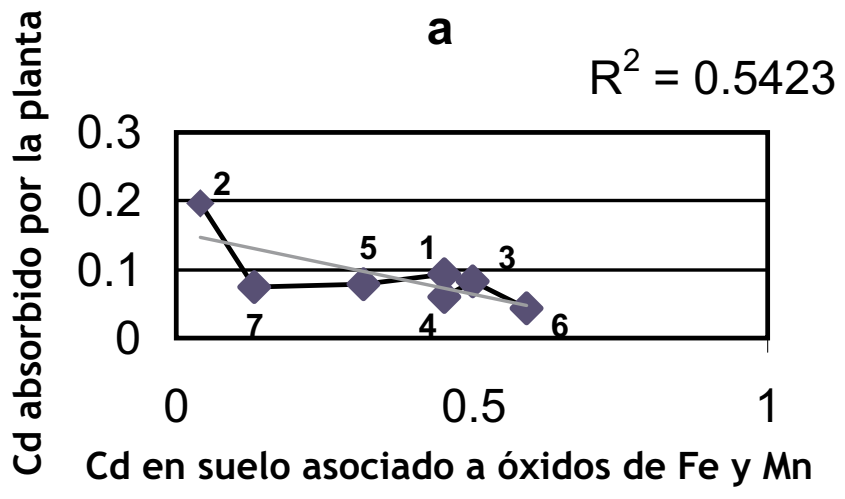

b

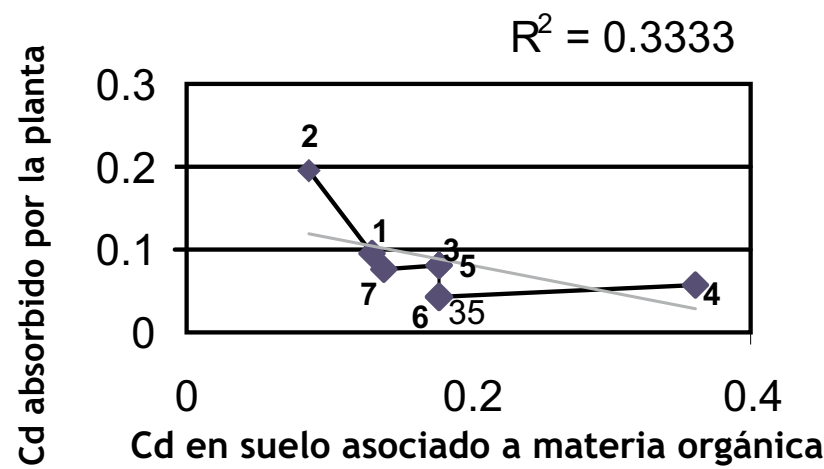

Figura 2. Relación entre el Método de Tessier. a. Cadmio asociado a óxidos de hierro y manganeso y la materia orgánica (mg/kg) y b. Cantidad de Cadmio absorbida por la planta $(\mathrm{mg} / \mathrm{kg})$.

con la textura de las muestras de suelo ya que se evidencia un alto contenido de arcillas y limo, lo que explicaría la acumulación de dichos metales. Las partículas de arcilla presentan valores elevados de superficie específica y capacidad de cambio catiónico, esto sumado a los altos porcentajes de materia orgánica, juega un papel importante en la retención de metales traza.

Según los datos de la Tabla 2, los niveles de Cd y As presentes en las plantas son altos (Cd $0.044-0.197$ y As $0.116-0.199 \mathrm{mg} / \mathrm{Kg}$ ). Según la OMS el consumo humano diario no debe exceder los $0.025-0.040 \mathrm{mg} /$ Kg., pues una ingestión mayor llevaría a la acumulación de estos metales en órganos como el hígado y riñones, que pueden afectar su funcionamiento $(7,8)$.

Se puede anotar que en el suelo 2 la relación entre el Cd extraíble del suelo y el absorbido por la planta es mayor que en los otros suelos. Lo anterior se justifica por los mayores contenidos de arcilla y el menor valor de $\mathrm{pH}$ registrados en la Tabla 1 . Es importante anotar que no hubo relación con los contenidos de $\mathrm{Cd}$ en la planta cuando la concentración en el suelo se determinó por el método convencional (contenido total).

Para As no se obtuvo relación con ningún método, lo que puede explicarse por las diversas propiedades y características de los suelos o por su manejo, pues resulta aventurado conocer sobre cuál es la fracción extraída del suelo con mayor afinidad a las concentraciones absorbidas por plantas.

Teniendo en cuenta los resultados obtenidos en esta investigación, se puede concluir que la concentración total de $\mathrm{Cd}$ y As en suelos no refleja la potencialidad de que el metal sea absorbido por la planta. En este trabajo las concentraciones de $\mathrm{Cd}$ y As absorbidas por las plantas sobrepasan los límites permitidos por la OMS, aunque los factores de movilidad de estos elementos son bajos, pero representan un riesgo medio para los cultivos que se efectúen en estos suelos.

Además, la disponibilidad y la movilidad del Cd y As no deben considerarse siempre como factores reales, sino de manera potencial, ya que dependen de las condiciones ambientales, del $\mathrm{pH}$ y del porcentaje de materia orgánica, entre otros. Los métodos estudiados para extraer el Cd y As considerados biodisponibles no demostraron correlación con las concentraciones absorbidas por las plantas, excepto el Cd extraíble con $\mathrm{Ca}\left(\mathrm{NO}_{3}\right)$.

La materia orgánica y los óxidos de Fe y $\mathrm{Mn}$ favorecen la retención de Cadmio en el suelo y disminuye su absorción por las plantas. De otro lado, es importante decir que los suelos aledaños a la cuenca del Río Bogotá sufren contaminación con metales pesados, adquirida principalmente por las aguas de riego, hecho que es corroborado por la presencia de $\mathrm{Cd}$ y As total en concentraciones considerables fitotóxicas.

Para finalizar, se sugiere seguir realizando investigaciones usando los mismos procedimientos y métodos utilizados en este estudio, pero teniendo en cuenta variables como: la cantidad de materia orgánica, el $\mathrm{pH}$, la textura y en general las características químicas del suelo. Además, es importante investigar sobre herramientas técnicas o sustancias que tiendan a minimizar la absorción de Cadmio y Arsénico por cultivos y pastos, así como identificar y controlar las fuentes de contaminación de la cuenca del Río Bogotá y sectores aledaños. 


\section{Referencias}

1. Vélez H. Impacto de los residuos industriales. Colombia. 1997.

2. Shukla S, Syers J. Amstrong D. Arsenic interference in the determination of inorganic phosphate in Lake sediments. J Environ Qual. 1972;1:292-295.

3. Aller A, Bernal JL, Del Nazal M. Geoquímica de los elementos traza. Instituto de Investigaciones Agrarias. Serie Tecnología Agraria $1989 ; 18,23-24$

4. Cala R. Metodología analítica en el estudio de metales pesados en suelos. Instituto Geominero de Espańa. Madrid. 1995.

5. Shi Y, Ruan J, Ma L, Han W, Wang F. Accumulation and distribution of arsenic and cadmium by tea plants. J Environ Biol. 2007;28 Suppl 2:493-502.

6. González S, Mejía L. Contaminación por cadmio y arsénico en suelos y hortalizas en un sector de la cuenca del río Bogota. Rev Suelos Ecuatoriales. 1995;25:51-56.

7. Organización mundial de la salud. Informe Cadmio. Ginebra: OMS. 1972.

8. Organización mundial de la salud. Informe Arsénico. México: OMS-OPS. 1987.

9. CORPOICA-TIBAITATA. Metodologías analíticas para la caracterización del suelo. 1986.

10. Chang CY, Wang CF, Mui DT, Chiang HL. Application of methods (sequential extraction procedures and high-pressure digestion method) to fly ash particles to determine the element constituents: A case study for BCR 176, J Hazard Mater. 2008. In press.
11. Kabata A. Trace elements in soils and plants. CRC press Boca Raton. FL. 2000.

12. Kabata A, Mukherjee A. Trace Elements From Soil to Human Ed. Springer. New York. 2007.

13. Anderson A, Hahlin M. Cadmium effects from phosphorus fertilization in field experiments. Swed J Agric Res 1981;11:2-6.

14. Arsénico y agricultura: Atender el problema de la "intoxicación masiva más grande en la historia” CIMMYT Febrero, 2004.

15. Fernández M. Arroz contaminado por arsénico en Estados Unidos publicado en: http://www.consumaseguridad.com/ciencia-ytecnologia/2005/08/30/19809.php.

16. Shukla S, Syers J, Amstrong D. Arsenic interference in the determination of inorganic phosphate in Lake sediments. J Environ Qual. 1972;1:292-295.

17. Mater N, McLaughlin, M, Heap M, Butt, M, Smart,M.(2002) Effect of current-season application of calcitic lime and phosphorus fertilization on soil $\mathrm{pH}$, potato growth, yield, dry matter content, and cadmium concentration. Commun Soil Sci Plant Anal. 2002; 33:2145-2165.

18. Ahsan DA, Delvalls TA, Blasco J. Distribution of Arsenic and Trace Metals in the Floodplain Agricultural Soil of Bangladesh. Bull Environ Contam Toxicol. 2008. In press. 